\title{
ITINERANCY OF QUASIEQUILIBRIA IN ONE-DIMENSIONAL GRAVITATING SYSTEMS
}

\section{T. TSUCHIYA}

National Astronomical Observatory, Mitaka, 181, Japan

N. GOUDA

Department of Earth and Space Science, Osaka University, Toyonaka, 560, Japan

AND

T. KONISHI

Department of Physics, Nagoya University, Nagoya, 464-01, Japan

One-dimensional self-gravitating many-body systems consist of $N$ identical parallel sheets which have uniform mass density $m$ and infinite in extent in the $(y, z)$ plane. We call the sheets particles in this paper. The particles are free to move along $x$ axis and accelerate as a result of their mutual gravitational attraction. The Hamiltonian of this system has a form of

$$
H=\frac{m}{2} \sum_{i=1}^{N} v_{i}^{2}+\left(2 \pi G m^{2}\right) \sum_{i<j}\left|x_{j}-x_{i}\right|,
$$

where $m, v_{i}$, and $x_{i}$ are the mass (surface density), velocity, and position of $i$ th particle respectively.

This system has been supposed to have three stages of evolution. (1)A system in a non-virial equilibrium experiences the violent relaxation and settles into one of the virial equilibria. (2) Small fluctuations of mean potential change energies of individual particles and the system tends toward equipartition, but the global shape does not change in this phase. (3) Collisions among the particles take place and the global shape changes toward the thermal equilibrium. Some authors suggested the existence of the three stages(Luwel and Severne, 1985; Sevene and Luwel, 1986), but we were the first to find the above evolutions numerically(Tsuchiya et al., 1994). We call the relaxation processes which take place in the second and the 
third stage, the microscopic and macroscopic relaxations, respectively. In the second paper(Tsuchiya et al., 1995) we found the time scales of the two relaxation: $T \sim N t_{c}$ for the microscopic relaxation and $T \sim 4 \times 10^{4} N t_{c}$ for the macroscopic relaxation, where $N$ is the number of particles and $t_{c}$ is the crossing time.

In usual gas dynamics, there is a unique relaxation, thermalization, and its mechanism is the diffusion of the phase point in the phase space. We found all the microscopic processes which happen in the microscopic relaxation shows the evidences of thermalization except for the fact that the global distribution does not change. Thus we continue our investigation to clarify what happens in the microscopic and macroscopic relaxations.

We studied the state of the thermal equilibrium in detail, and found the thermal equilibrium is defined as a time average of different quasi-equilibria, over hundreds of the macroscopic relaxation time, $T_{\text {macro. }}$. In a time scale of $T_{\text {macro }}$, the system changes the state from one quasi-equilibrium to another. From the fact we can surmise the phase space dynamics as follows.

1. The microscopic relaxation. It is a diffusion driven by mean field fluctuations. It takes place on the time scale $T_{\text {micro }} \sim N t_{c}$. This kind of diffusion is also studied by Miller (1991).

2. Quasi-equilibria. The diffusion of the microscopic relaxation is restricted in a region for a long time, then the orbit in the phase space travels all over the region. Hence the system shows the "mock" ergodicity. Its lifetime is $4 \times 10^{4} N t_{c}$ for the water-bag distribution, for example.

3. The macroscopic relaxation. After long time, the orbit escapes from the quasi-equilibrium region. This happens when the orbit find a small window to the outside of the wall which restricts the orbit in the region. Then the orbit visits all the regions in the phase space, the system attains the thermal equilibrium.

The detailed analyses will be published elsewhere.

\section{References}

Luwel, M. and Severne, G. (1985) Collisionless mixing in 1-dimensional gravitational systems initially in a stationary waterbag configuration, Astron. Astrophys., Vol. 152, pp. 305.

Miller, B.N. (1991) Gravity in One Dimension: Diffusion in Acceleration, J. Stat. Phys., Vol. 63, pp. 291.

Severne, G. and Luwel, M. (1986) Violent relaxation and mixing in non-uniform onedimensional gravitational systems, Astrophys. and Space Sci., Vol. 122, pp. 299.

Tsuchiya, T., Tetsuro, K. and Gouda, N. (1994) Quasi-equilibria in one-dimensional selfgravitating many body systems, Phys. Rev. E, Vol. 50, pp. 2706.

Tsuchiya, T., Gouda, N. and Tetsuro, K. (1995) Relaxation processes in one-dimensional self-gravitating many-body systems, Phys. Rev. E, submitted. 